\title{
Towards a framework for the elicitation of dilemmas
}

\author{
Marc J. C. Burger
}

Published online: 8 August 2007

(c) Springer Science + Business Media B.V. 2007

\begin{abstract}
This paper covers the main findings of the doctoral research that was concerned with seeking to extend aspects of dilemma theory. In professional practice, the Trompenaars Hampden-Turner Dilemma Reconciliation Process ${ }^{\mathrm{TM}}$ is a vehicle delivering dilemma theory in application. It informs a manager or leader on how to explore the dilemmas they face, how to reconcile the tensions that result, and how to structure the action steps for implementing the reconciled solutions. This vehicle forms the professional practice of the author who seeks to bring more rigor to consulting practice and thereby also contribute to theory development in the domain. The critical review of dilemma theory reveals that previous authors are inconsistent and variously invalid in their use of the terms 'dilemma theory,' 'dilemma methodology,' 'dilemma process,' 'dilemma reconciliation,' etc., and therefore an attempt is made to resolve these inconsistencies by considering whether 'dilemmaism' at the metalevel might be positioned as a new paradigm of inquiry for (management) research that embodies ontological, epistemological, and methodical premises that frame an approach to the resolution of real world business problems in (multi) disciplinary; (multi) functional and (multi) cultural business environments. This research offers contributions to knowledge, professional practice and theory development from the exploration of the SPID model as a way to make the elicitation of dilemmas more rigorous and structured and in the broader context of exploring 'dilemmaism' as a new paradigm of inquiry.
\end{abstract}

Keywords Dilemma $\cdot$ Methodology $\cdot$ Elicitation $\cdot$ Reconciliation $\cdot$ Paradigm 


\section{Introduction}

\subsection{The origins of this research}

The main agenda for this research arose from the author's career as a management consultant during which I became increasingly concerned to improve my professional practice by engaging extant theory and thereby seeking more rigor of 'knowledge in application.' In developing exploratory papers in the early stages of my research, I soon realized weaknesses in my professional practice and identified significant gaps in knowledge in the literature. The overall ontology of my employers consulting company is based on forcing the consideration of opposites as a new paradigm of inquiry for consulting. Undertaking research in this area has considerable implications not only in terms of the formal theory base but as presented in the body of this paper; the inquiry paradigm offers a new alternative approach for how to conduct research in a wide range of business and management issues.

Over recent decades we witness a major change in the world of work. Power is diffused and shared. In contrast with traditional management, where structures and systems are derived from a pre-defined strategy, the new workplace is seeking to balance what matters for the company (its strategy) and what matters for the individuals (their life strategies). In fact, management and employees decide and execute inter-actively. In this New World of the customized workplace in which priority for sustained personal development goes hand-in-hand with the employer's business performance and growth, the reconciliation of dilemmas is the new source of authority. This is revealed even more dramatically in the process of continuing globalization. New approaches are required to elicit the dilemmas that these changes generate and to offer solutions.

The intention is to inform and improve professional practice and to contribute to the theory development in this domain. In the early stages of my research, the researcher had undertaken a preliminary investigation of how different consultants in my peer group analyzed responses from eighteen participants of one of our clients. It revealed that different consultants when faced with the same source data draw varying conclusions. Although the client might have been satisfied with any one of the conclusions from any one consultant, this showed clearly the absence of rigor and objectivity.

As a result of these early insights, this research became guided by two main research questions:

(a) What are the dynamics in the process of seeking to explore and elicit dilemmas?

(b) Can the elicitation of dilemmas be made more systematic and rigorous through the development of supporting substantive theory?

The research seeks to extend existing knowledge by examining the process of the elicitation of dilemmas and how this might be made more rigorous within a theory base. The existing body of knowledge reveals that dilemma thinking derives from many sources. Its origins can be traced to philosophy (Russell 1959; Kant 1996), human psychology (Jung 1971; Fromm 1956), language (Saussure 1960), anthropology (Lévi-Strauss 1963; Bateson 1972, 1976), existentialism (May 1967; Frankl 1978; Camus 1991; Sartre 1993), human psychology (Benedict 1934; Maslow 1954), organizational behavior studies (Blake and Mouton 1985; Trist and Emery 1990), leadership 


\section{Hampden-Turner and Trompenaars framework to reconcile dilemmas}

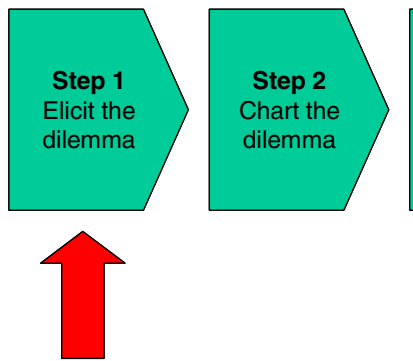

Gap in knowledge
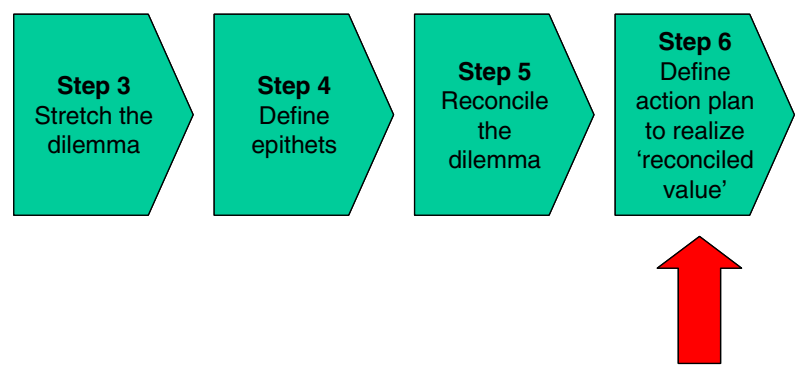

Gap in knowledge

Fig. 1 Identified gaps in knowledge in THT Consulting dilemma reconciliation process

(McGregor 1987), strategic planning (Mintzberg 1994; Porter 1980), organizational development (Cameron and Quinn 1999; Handy 1990) and many more fields.

There is also some literature available on reconciling dilemmas; examples are Hampden-Turner and Trompenaars (2000), Johnson (1992), and De Wit and Meyer (2000). However, all this literature ignores the problem of identifying the 'substantive' and/or 'relevant' dilemmas in the first place. And when a multiplicity of dilemmas is found (as is usually the case in real-world client assignments), no literature could be found for prioritizing these dilemmas - thus which dilemmas should be reconciled first and/or which will have the more significant benefits once reconciled? In addition, existing knowledge does not address with any theory basis how the implementation of the reconciled solutions might be structured.

In their consulting work, Trompenaars Hampden-Turner (THT Consulting) use a six-step approach for facilitating their interventions with clients centred around expressing client problems as dilemmas, codifying these dilemmas in a standard format, and then finding solutions (reconciliations) to these dilemmas. Steps 2 through 5 are well verified and validated through both research and practice and have been published in both the professional and formal academic press. Step 1 in particular is less rigorous in practice because as mentioned above it suffers the absence of a theory base. Step 6 is also less defined and less rigorous Fig. 1.

The research described in this paper focuses on the former, i.e., Step 1.

\subsection{Terminology, initial tautology, and design considerations}

It should be noted that the literature (and most of the recent doctoral theses in this subject are) variously and confusingly use many of the terms to describe this whole discipline inconsistently. Thus, the area is sometimes described as dilemma theory (Hampden-Turner and Trompenaars 2000), dilemma methodology, dilemma analysis, dilemma reconciliation process (or DRP), dilemma method, polarity management (Johnson 1992), and paradox management (Quinn and Cameron 1988; de Vries 1995; de Wit and Meyer 2000), etc.

For reasons that are discussed in the body of this paper, the author has chosen to try to clarify and position the whole domain by adopting the stance that the conceptual framework can benefit by being described as 'dilemma-ism' or 'dilemmaism.' 
By dilemmaism is meant an exploration of a (new) paradigm of inquiry as the meta-level construct of dilemma theory. This paper gives the opportunity to explore the ontological, epistemological, and methodological components of dilemmaism.

At this point, there is a tautology. At the meta level, this 'research' is about 'researching' a way of doing research and therefore it is neither appropriate nor possible to follow convention and describe at this point the basic methodological choice for the research, because the research itself is a challenge to established methodological approaches. Dilemmaism includes the (new) methodology of dilemmaism! The approach to the research lies in the exploration of dilemmaism and indeed the methodology within dilemmaism. At this stage of the research, methodological terms are therefore deliberately used loosely and flexibly.

To enable the debate to proceed the apparent 'pick and mix' approach to using different research methods was not ad hoc or eclectic, but chosen to collect primary data from which the methodology within dilemmaism could itself be clarified. This is subject to a rigorous explanation in Chap. 2 and resolved before the end of this paper.

The research was conducted within THT Consulting as the host environment and four of their major clients. The investigative stages of the research were carried out primarily during the period December 2001 and April 2004. In collecting research evidence, surveys with prescribed closed questions were avoided, as they would otherwise induce phenomena understood from the researcher's own perspective and that of the other internal consultants within THT Consulting. Therefore, the research adopts a qualitative inter-actionist approach allowing the exploration of different means to elicit dilemmas using methods that include participant observation, semi-structured interviews, as well as using existing interview transcripts and online interviews.

However, quantitative approaches were used to analyze the interview transcripts to seek a more rigorous and systematic exploration of this data and maximize what could be inferred and made from it. In the second phase, the investigation of models for eliciting information used in other professional practices was collected via both semi-structured interviews and literature research. In the final phase, the modification and application of an adapted model that sought to make the process of eliciting dilemmas more rigorous was tested via semi-structured interviews.

\section{Methodological issues in dilemmaism}

This chapter serves to consider the methodological aspects of dilemmaism. The discussion considers the methodological choices faced by the author in developing the research design but is necessarily extended because of the very nature of dilemma theory and dilemmaism.

Since the 1962 publication of The Structure of Scientific Revolutions by Kuhn (1970), the term paradigm has been widely used by social science researchers. It is generally synonymous with philosophy, as paradigm refers to the way of looking at phenomena, at first principle or ultimate, a worldview, or a perspective from which distinctive conceptualizations and explanations are made (Gill and Johnson 2002). It is the underlying philosophy and assumptions about the world and the nature of knowledge, a framework of theories, methods and interpretations or a meta-theory. Its importance to the researcher is that paradigm suggests how the research should be conducted, how data are collected and analyzed, and even writing of the results. 
In general the two major approaches to research are: quantitative, which is a positivist approach; and, a qualitative, the more interpretive approach. Some authors also use the term phenomenological instead of interpretive (Easterby-Smith et al. 1991). These two approaches are also referred to as deductive or inductive respectively (Silverman 1993; Mason 1996; Hussey \& Hussey 1997; Neuman 1997; Seale 1998; Creswell 2003).

Positivism is an organized method of combining deductive logic with precise empirical observations on individual behavior. This, in order to discover and confirm a set of probabilistic causal laws that can be used to predict general patterns of human activity (Neuman 1997). Positivism questions the existence of chaos. In the eyes of positivists the world is ordered, logical and predictable. The basic nature of human beings to a positivist is that they are self-interested, pleasure seeking and rational. They operate in a way that reacts to external causes, and each individual reacts in the same way to the same causes. For example Durkheim (1947) noted, "Social phenomena are things and ought to be studied as things" (p. 27). The positivist belief in and rely on the evidence of external reality suggests that there is no need to examine the unseen, internal motivations of an individual's behavior. Positivism has been the dominant paradigm although there is a continual debate marked by misconceptions over the literature (Hunt 1991).

The interpretive view of research is concerned with, and how the social world is interpreted, understood or experienced. Denzin and Lincoln (1994) describe qualitative research as being multi-method in focus involving an interpretive, naturalistic approach to the subject matter. Neuman (1997) points out that, in contrast to positivism's instrumental approach, the interpretive method adopts a more pragmatic orientation involving a practical examination of how people manage their affairs. This approach involves long-term observation of day-to-day ways that people use to make sense of their world and get things done. Wolcott (1975) notes that one of the most significant characteristics of qualitative research is that the researcher is pre-eminently the research tool.

A methodology that comes between the two extremes of positivism and interpretivism is Critical Social Science or 'mixed methods' (Creswell 2003). Critical researchers have a fundamental goal, that of attempting to change the world. Whereas the interpretivist approach to research involves trying to understand social life and importantly, how people give meaning to their surroundings, the critical researcher attempts to uncover myths and revealing truths thereby enabling them to change their lives. According to Newman and Benz (1998) the research situation today is less 'quantitative versus qualitative' and more how research practices lie somewhere on a continuum between the two extremes. Creswell (2003) claims that the best that can be said is that studies tend to be more quantitative or qualitative in nature. Consequently, a variety of methods and approaches are combined in this methodology.

As a researcher I was faced with my own methodological dilemma! Qualitative inductive research based on methods like semi-structured interviews, observation, etc. produces rich outputs that have high validity. However, the reliability may be low if these findings cannot be transferred from a small sample to a wider population. By contrast, findings from quantitative research based methods, like questionnaires, can be made highly reliable by careful selection and structuring of the sample. However, such reliability, on is own, does not mean that the findings are valid. 


\subsection{This OR that, this AND that?}

There is a fundamental challenge that can be made to all the above. It would appear that the reader should want to know. Which of the alternatives did the researcher select? Or did he/she select a mix of the (traditional) options? Thus, did the research follow a singly deductive OR inductive approach? If not, then it 'must' be presumed that the research was deductive AND inductive (mixed approach). Did the research follow a singly positivistic OR constructivistic approach? If not, then it 'must' be presumed that the research was both positivistic AND constructivistic.

The reader (and researcher) are locked in to this way of thinking because they are bound to Boolean or Cartesian Logic. As Trompenaars and Woolliams (2003) explain, these approaches summarised by Heron and Reason (1997), Creswell (1998, 2003) and others are based on western thinking. Most of these debates owe their origin to US American and European writers where these approaches to research have been developed. Other (more eastern) cultures are not bound by this Cartesian Logic ('OR' 'AND' 'NOT').

Dilemmaism opens up an entirely new belief system and therefore a new way of thinking about research. It begins with the fundamental notion of adding 'AND' to opposites and begins the process of reconciliation. Dilemmaism is built upon THROUGH thinking.

Thus traditionally (under Cartesian logic) the researcher asks:

- Shall I follow a deductive OR inductive approach?

- Shall I follow an approach, which is partly deductive AND inductive?

With such thinking, it would appear that there are no other alternatives. Under dilemmaism, the researcher can also ask:

'THROUGH using initially a deductive approach, how can I identify how I can follow with an informed inductive approach? Then THROUGH using this inductive loop, how can I identify how I can follow with an informed deductive step?'

Thus, within dilemmaism the findings (for example) semi-structured interviews serve as input to structure (for example) a questionnaire. And conversely, the feedback from questionnaires can be used to define the questions to be asked in semi-structured interviews. Consequently a continuous learning loop is created, see Fig. 2.

The methodology of dilemmaism can be stated therefore as the reconciliation between an inductive and a deductive paradigm of inquiry.

Whereas, 'mixed methods' could be perceived as the compromise between the two. Doing both semi-structured interviews and questionnaires independently, solely for triangulation, which is the case with mixed methods, is not enabling a continuous learning loop (Trompenaars and Hampden-Turner 2001). The reconciliation of this dilemma, inductive versus deductive is demonstrated in Fig. 3.

\subsection{Dilemmaism}

Thus dilemmaism can be seen as a new paradigm of inquiry. In other respects, it is more than 'simply' adding a new alternative to the list of alternative inquiry paradigms because it adds much more than just adopting a 'pick and mix' approach to undertaking research. 


\section{Deductive approach}

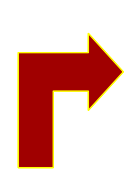

Apply universal, deductive principles to ensure reliability and generalisibility, although ...

\begin{tabular}{|c|}
\hline We do not want to drown \\
in particular situations or \\
lose our sense of general \\
propositions ...
\end{tabular}

Deductive approach with inductive adaptations and discretion and vice versa
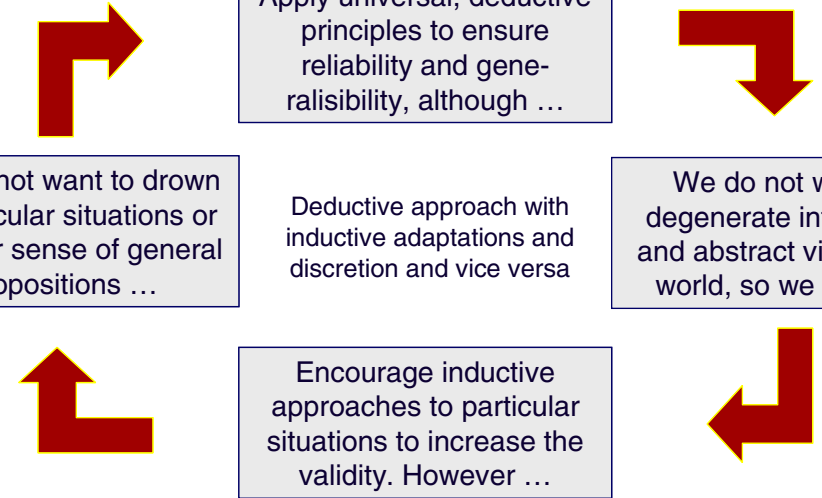

We do not want to degenerate into a rigid and abstract view of the world, so we must ...

\section{Inductive approach}

Fig. 2 Dilemmaism's continuous learning loop

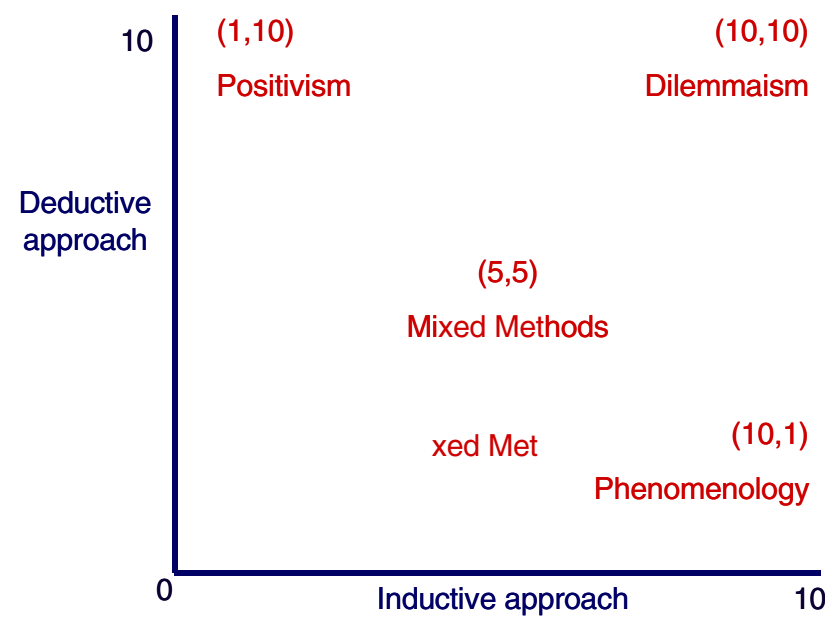

Fig. 3 Dilemmaism: inductive versus deductive reconciled

For business applications, dilemmaism has the potential to overcome the limitations of traditional approaches to inquiry such as positivism, constructivism, postmodernism and mixed methods, which do not take account of competing or contrasting values that are central challenges to modern organizations. As a paradigm, the components of dilemmaism can be summarized under the following:

\subsubsection{Dilemmaism ontology}

The ontology is based on a language, associated representational vocabulary, and prescribed analytical steps that codifies, defines, categorizes, and resolves dilemmas. The central construct is that in today's business world, organizational, managerial; and leadership issues can be captured and thereby made explicit as a tension between two 
(desirable) opposites (Cameron and Quinn 1999). The ontology defines dilemmaism as the basis for eliciting the business problems in the investigation of this paradigm in order that a process of reconciliation can accommodate the seemingly contrasting alternatives.

\subsubsection{Dilemmaism epistemology}

The epistemology is both pragmatic and memetic. Today's organizations need stability and change, tradition and innovation, public and private interest, planning and laissez-faire, order and freedom, growth, and decay (Trompenaars and Woolliams 2003). These dynamics deriving from economic and societal changes that include globalization, increasing diversity and ever pervading of technology mean those traditional epistemologies are insufficient to accommodate competing values simultaneously. The consequence is that the systems and processes of organizations are changing to a world of dilemmas.

\subsection{Research design}

One might also use the underlying construct within dilemmaism as a means to reflect on the research process! Thus, dilemmaism can in part be viewed as the reconciliation between the deductive versus inductive approach. Overall, the research design consisted of three research cycles:

(1) In the first research cycle an investigation was centered on the current practice of eliciting dilemmas using a qualitative approach intended to generate rich and valid outcomes.

(2) The second research cycle used semi-structured interviews for exploring best practice in eliciting information in other professional disciplines as a basis for considering what lessons may be learnt and transferred to the elicitation of dilemmas in management consulting.

(3) A new framework for eliciting dilemmas was conjectured from research cycles 1 and 2 based on an established model used for sales management and this was explored and tested in working professional practice.

Note that the positions mentioned in the grid (Fig. 4) are indicative.

\section{Research cycle one}

In this first research cycle, current practice in the way that THT Consulting tries to elicit dilemmas faced by clients was explored. A number of experiments described below were performed.

Observing how a senior consultant would conduct interviews with senior managers. A senior colleague has conducted several interviews at major financial institution. During six interviews the researcher has observed him to find out what kind of key interview tactics he was using in order to tease out the dilemmas the client was facing (Table 1). 


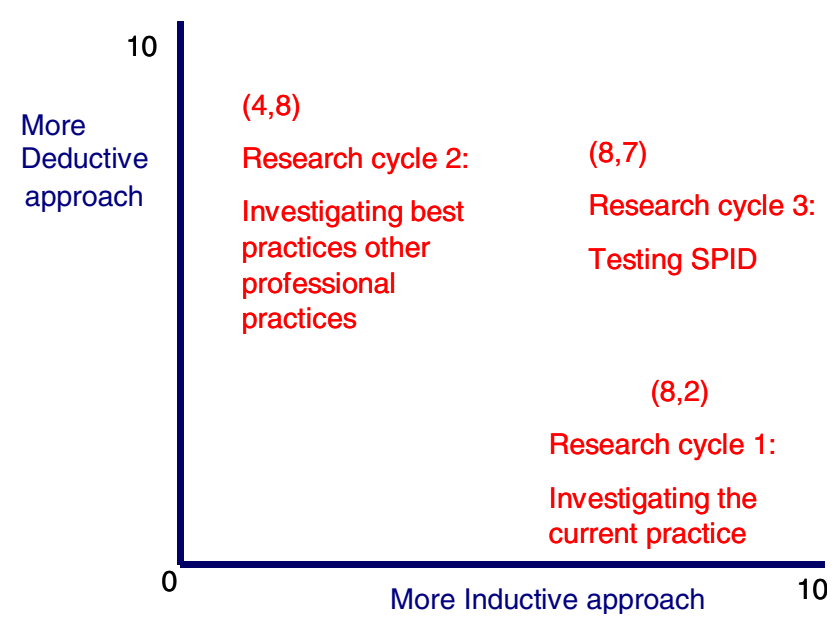

Fig. 4 Positions of the three research cycles on the deductive versus inductive dilemma grid

Table 1 Strategies for eliciting dilemmas (Source: Hampden-Turner 1999)

\begin{tabular}{ll}
\hline Interview strategies used by peers for eliciting dilemmas & Applied \\
\hline $\begin{array}{l}\text { Take a value to the extreme. The interviewer on purpose exaggerates the value so that } \\
\text { it turns into pathology }\end{array}$ & No \\
$\begin{array}{l}\text { Focus on the opposing value. The interviewer stipulates the importance of value on the } \\
\text { other side of the continuum }\end{array}$ & Yes \\
$\begin{array}{l}\text { Focus on personal conflicts. The conflict within each person has become a conflict } \\
\text { between persons each at one horn of the dilemma }\end{array}$ & Yes \\
$\begin{array}{l}\text { Focus on the condemnation of indecisions. The interviewee is belittling of those } \\
\text { distressed by the conflict }\end{array}$ & Yes \\
$\begin{array}{l}\text { Gauging the centrality of key issues and the amount of emotional energy invested in } \\
\text { key dilemmas }\end{array}$ & No \\
$\begin{array}{l}\text { Testing the validity of what the first interviewee said with the second, third and nth } \\
\text { interviewees to see if there were common convictions. (Analytic Induction) }\end{array}$ & Yes \\
$\begin{array}{l}\text { He tested the acceptability of possible solutions to find out if he was way off the } \\
\text { underlying issues }\end{array}$ & Yes \\
\end{tabular}

\subsection{International leadership study}

As part of an international leadership study a series of interviews were conducted with the objective to gain insight in the characteristics of effective leaders who had (recently) gone through a merger or alliance process or made a substantial take over and the dilemmas they were facing. As part of this research 13 interview transcripts were used. NUD*IST was used as a vehicle for handling and coding these interviews. Coding was applied to record what kind of transaction occurred for each (identifiable) interaction that is interactions deriving from both questions by the interviewer and answers by the interviewee. 


\subsubsection{Transactional analysis (TA)}

Transactional Analysis (TA) is as a system that seeks to understand the interactions of people and to improve the human social environment. Berne (1964) identifies three different 'ego states': Parent (language of values), Adult (language of logic and rationality), and Child (language of emotions). The researcher has looked for each interaction, both question from the interviewer and the answering by the interviewee, and thereby to code what kind of transaction occurred. Given the three different 'ego states' within TA that results in total of nine combinations of transactions are possible. The factual findings revealed:

(1) The 'set up' (start) of the interviews had only 'Adult' questions.

(2) Tensions during the interview (or crossed interactions) are needed to come to the elicitation of dilemmas, mainly 'Adult-Parent.'

(3) Once a dilemma is elicited and discussed the conversations has a tendency to slip into a complimentary transaction, mainly 'Child-Parent.'

\subsubsection{Neuro linguistic programming (NLP)}

Neuro Linguistic Programming (NLP) began as a model of how we communicate to ourselves. This model explains how we process the information that comes into us from the outside (Bandler and Grinder 1975). Following the same rationale as with TA, the same 13 interviews were coded from an NLP perspective. The text was examined for each sensory input channel of both the interviewer and the interviewee. The factual findings revealed:

(1) six of 13 have build up rapport (= complementary transaction); 7 of 13 not.

(2) No linkage of any significance could be identified between 'rapport' or 'no rapport' and the process of the elicitation of dilemmas.

\subsubsection{Corporate culture}

Since culture is about meaning, and communication is an expression of meaning, the language used by people in organizations is also indicative of organization culture. Watt (2002) has developed mechanisms for determining indicators of cultural profiles by a linguistic analysis of such language using Trompenaars' Corporate Culture model (1998). The factual findings revealed:

(1) All (13) interviewees and interviewers used predominantly 'incubator' language.

(2) Interviewers had predominantly 'guided missile' as second vocabulary.

(3) The same 'Corporate Culture language' creates rapport; however this loses the opportunity to challenge with the opposing values to stimulate debate.

(4) Interviewees tend to focus on more ideographic dilemmas, rather then nomothetic.

(5) There is evidence that people use language of the desired (corporate culture) situation rather than the current situation.

\subsection{Categorizing raw dilemmas}

How different consultants from within THT Consulting might interpret raw data and draw conclusions in the form of a series of (client) dilemmas. I have researched a real 
live example of 18 participants of a client who filled in their dilemmas in a system based on interactive web pages. I have investigated the categorization process of clustering raw participants output into key-dilemmas from three different perspectives, namely my own point of view, the experience of six different colleagues and the experience of two representatives of the client. Listed below is an analysis of the different approaches consultants and clients used when categorizing dilemmas together with a comparison of these approaches. The factual results give rise to a number of issues that indicates the complexity of categorizing raw dilemmas.

- It was found that different users in different situations generated a differing number of dilemmas, i.e., no unique re-occurring number or description of dilemmas was delivered. In addition there was variation in the use of models.

- It was also found that 'a' (or 'the') important dilemma might be missed all together. If a certain raw dilemma is mentioned only once or twice one might run the risk that it is left out in the categorization, while it can be very important for the organization.

- It was found that all consultants and the two clients raised the issue that dilemmas proposed differ considerably in 'quality.' Subjects had to a certain extent to imagine or discern what was meant by the raw dilemmas, because the meaning of the dilemma was not clearly conveyed in the explicit summary text that described the raw dilemma.

- The 'cocktail party effect' is the tendency to focus ones listening attention on a subject of particular interest from a single talker among a cacophony of conversations and background noise (Arons 1991). To a certain extent this principle could also be applied for the process of categorizing raw dilemmas, in the sense that one or a few words jump out the list and stand apart for the rest because they relate in some way to the investigators interest.

\subsection{Conclusions from research cycle 1}

Resulting from this first research cycle, the following conclusions can be drawn:

(1) Based on current practice and methods, different consultants produce different dilemmas from the same source material;

(2) Experienced Consultants use their 'experience' and tacit knowledge, which works for them, but is difficult to transfer to junior consultants and importantly, cannot be transferred to the client (in order that they can embed Dilemma Reconciliation in their organization);

(3) 'Adult-Adult' or 'Child-Parent' interactions (that is the relationship between the consultant and interviewee) tend to conceal dilemmas;

(4) Both interviewers and interviewees used predominantly 'incubator' vocabulary, which induces an ideographic mindset for the interviewee whereas the problems of the client's organization are fundamentally nomothetic. Tensions between the Corporate Culture typologies are not exploited.

(5) No link could be identified between (NLP) 'rapport' or 'no rapport' and the process of the elicitation of dilemmas.

Overall this research cycle demonstrates that the existing methods and approaches used to elicit and capture dilemmas are not reliable or rigorous and that elicited dilemma statements too often appear to be invalid. It can be concluded that this is 
because they do not have any proven substantive theory basis. As a consequence, they are not providing the optimum service to the client because they are not correctly diagnosing the issue of inquiry correctly. What is needed is a more systematic and structured theory based approach.

\section{The investigative study for research cycle two}

Building on the weaknesses in the current practice of eliciting dilemmas, demonstrated in research cycle 1 , a new framework for eliciting dilemmas was constructed as part of the second research cycle. In this research cycle, different strategies, frameworks or other systematic approaches other professions apply for eliciting the knowledge from their respondents based on their agenda were also explored. To investigate these different disciplines, several interviews were conducted with respondents from different professional practices. Eight professionals were approached and interviewed, these were: a medical doctor, barrister, public prosecutor, system analyst, sales representative, domestic appliance service engineer, police detective, and a police psychologist.

The following generic questions were posed (although the actual wording used was adapted to the specific professional practice of each respondent).

(1) How do you elicit the issues? Please describe the process you follow?

(2) Were you trained on a specific method? If yes, which method?

(3) How do you know your intervention was successful in terms of achieving validity in establishing what you need to know?

(4) How would you describe the nature of the relationship you build with your clients?

A further literature review was conducted on how information is elicited in other professional practices as an input to this research cycle and to prepare for the interviews.

\subsection{Evaluating models used in differing professions}

Any dialogue is essentially based on posing questions. Totally random questioning (or even silence!) is of course un-structured and although appropriate to some kinds of therapy, is not a systematic deductive process that can converge to a definitive elicitation of the information sought. The literature indicates that many professions do appear to adopt some basic premise and follow a pre-defined rationale. A review of some common approaches reported was undertaken to see if any of these methods could be transferred or adapted to the elicitation of dilemmas and what new insights could be gained from such comparisons.

Whilst the different professions that have been researched have different agendas to the consultant seeking to elicit dilemmas as a basis for solving their client's business problems, a number of insights were gained and a number of inferences can be drawn. These help to inform the next cycle of research.

(1) It appears that most of the professionals receive some sort of basic model or framework when they receive their initial training to help them get started, but this more mechanistic process (which they can describe) becomes subsumed as they gain experience into a more tacit process (which they necessarily find much more difficult to describe). What is common is the benefit of having an initial framework that the professional can use as a starting point. 
(2) Another issue is the degree to which the respondent is intentionally collaborating with the professional to offer the information sought, or whether the respondent is trying to conceal or avoid or hinder the transfer of information to the professional. The business consultant can normally expect to be faced with a co-operating respondent-because the aim is to help the individual manager/ leader solve the business (nomothetic) or individual (ideographic) problems deriving from dilemmas in the work place. In what can be expected to be more rare cases, the consultant may be faced with respondents who are hostile. This may occur if the respondent feels their job or integrity is threatened by a re-organization, cost cutting or downsizing that may result from the intervention by the whole consulting assignment.

(3) Thirdly is the competence of the respondent is being able to describe accurately the symptoms of the problem and what data to include in their responses. Thus, the degree to which householders describe the problems of their central heating may vary from a generic 'it's not working' to 'after we have run a bath of hot water, no hot water is available from the kitchen but we can still draw off hot water in the bathroom.' Or 'this has been happening since last Friday when we had a power-cut in a thunderstorm.'

(4) Related to this is the competence of the respondent to be able to report what data is material to the case. Thus some householders may mention the thunderstorm while others not. Some patients will offer to tell their doctor they have just returned from a tropical country where the hygiene was poor. Similarly some managers may offer details about a recent business crisis and others not.

(5) There is the danger of 'seeding' where the professional gives an example or asks too specific questions and induces responses from the respondent that he/she would not otherwise have construed or offered. If the learned doctor says 'you might have an ulcer,' the patient may selectively describe some of their symptoms that fit with the diagnosis rather than offering other facts (like 'I am eating too much junk food').

(6) A more subtle issue is whether the respondent already knows what the problem is and can describe it correctly or whether it is only the professional who can diagnose the issue. Even worse, the respondent may claim they know the problem and distort the evidence but what they select to report and how they say it. For example, 'It's only indigestion doctor, can you give me some pills' - whereas the patient may have a more serious complaint.

Based on an evaluation, all the cases offer food for thought but the models used by the (some) sales representatives, the Huthwaite SPIN (Rackham 1988), merits further consideration. This model has elements, which are similar to the issues faced by the consultant in seeking to elicit dilemmas. Of the alternatives reviewed in this research cycle and what was also found in the literature, this model thus appears to offer some ideas and a basic framework that merits further investigation and research in application. For these reasons, it was concluded that the Huthwaite SPIN model should be explored as a basis for the subsequent part of this research cycle.

\subsection{Review and adaptation of the SPIN model as a basis for further experimentation}

The closure (final step) for the SPIN model in the selling situation is of course ' $\mathrm{N}$ ' (explicit needs). To apply an equivalent process for the elicitation of dilemmas this 


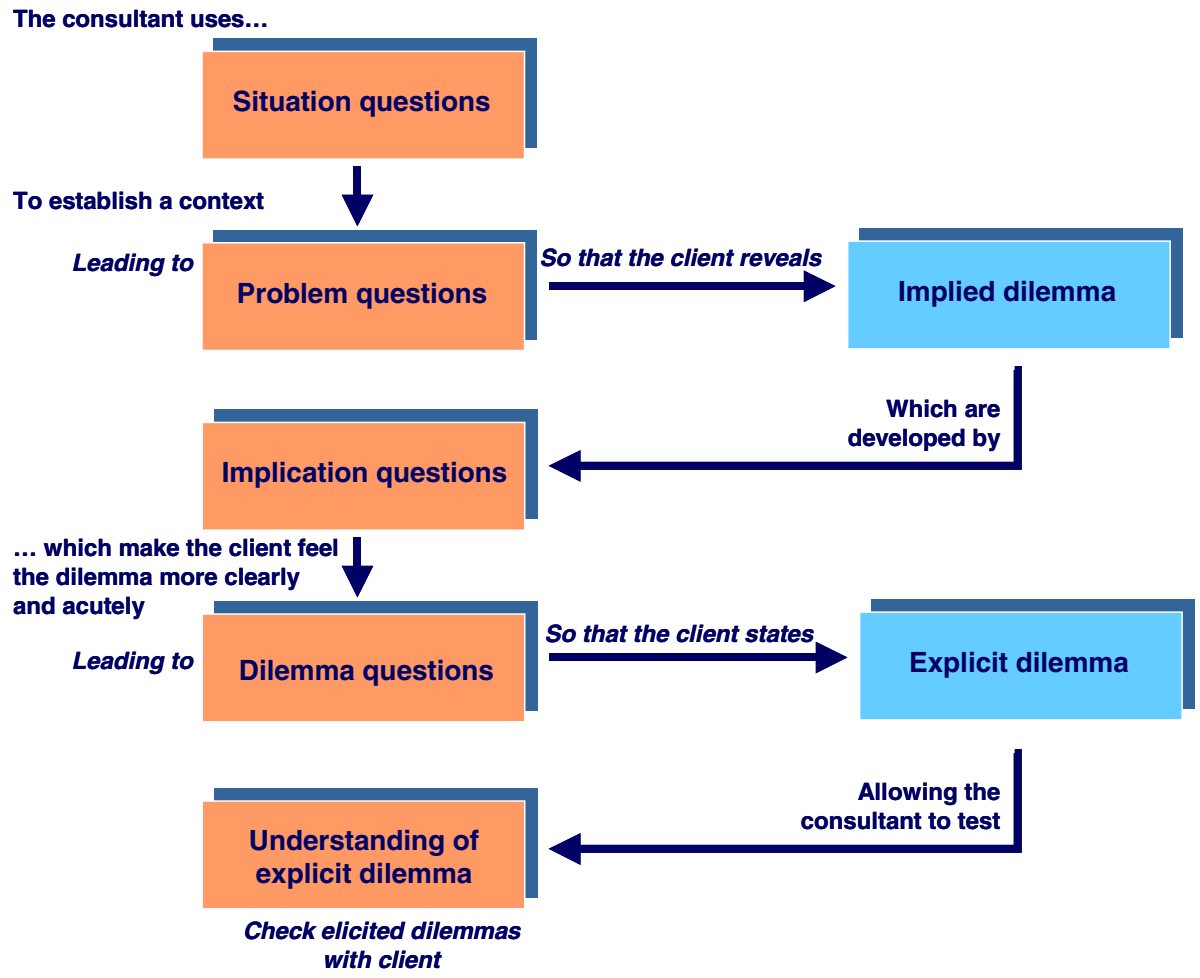

Fig. 5 SPID model

was modified to SPID where the closure is the elicitation of the dilemma(s). Thus, the underlying intent is to define and explore a structured framework based on this substantive model that can be used to provide rigor in a systematized manner and thus convert initial ideas, unstructured thoughts, and vague perceptions of issues offered by clients into strong, clear desires for reconciled solutions of explicit dilemmas. These structured questions follow the same rationale as the SPIN approach and form the basis of the dilemma elicitation model-SPID that is questions based on the paradigm 'Situation, Problem, Implication, and Dilemma.' It was conjectured that by probing the client problem in this structured format, issues could be explored in a more systematic way leading to a more objective elicitation of the true underlying dilemmas. Furthermore, this would appear to provide the starting point for training new consultants (and managers themselves) in a structured process for eliciting dilemmas. See Fig. 5. Research Cycle 3 was conceived to explore the effectiveness of an approach based on this proposed SPID framework.

Learning from the current research cycle, the steps and rationale of this proposed SPID model are now debated in the following section. As far as possible, the language and form of the original SPIN model is retained.

\section{Research cycle three}

In this research cycle, a further series of experiments were conducted in which the researcher was seeking to elicit dilemmas from a number of business managers/ 
leaders. In fact 'real world' consultancy assignment interviews were used, rather than 'laboratory' experiments. Thus, this research cycle was more ethnographic in nature. Instead of following the more established format of such interviews (based on current professional practice), the researcher operating in consultant mode, sought to apply and test the SPID model.

This research cycle was undertaken in two phases. In phase 1 the author (in the dual role of both researcher and consultant) and a peer consultant conducted a series of interviews using the SPID framework. The intent was to explore how the application of SPID enabled or facilitated the elicitation of dilemmas. In this phase a consultant, a peer of the researcher, and the researcher himself interviewed eighteen members of the Leadership team of a major client to the researcher's employing consultancy organization.

The researcher conducted seven interviews himself, a peer consultant undertook seven interviews and four interviews were conducted jointly. In all cases, the consultant/researcher applied the SPID model. The (peer) consultant and the researcher made individual (independent) notes. These notes were not shared between the consultant and the researcher until the end of the series to prevent one influencing the other. After the consultant and the researcher individually elicited dilemmas the results were compared. After the comparison, the supplementary interview notes were exchanged.

In phase 2, a senior consultant (3), studied all the interview notes made by consultant 1 and the researcher himself (consultant 2). In addition he conducted two additional interviews at the client side: with the project sponsor and the project leader. Furthermore, he conducted interviews with both consultant 1 and the researcher (consultant 2). Based on these inputs, consultant 3, constructed a revised list of key dilemmas for the client. Note that this consultant (3) did not use SPID, but he is very experienced in the elicitation of dilemmas. A few weeks later, a workshop was conducted with consultant 3 and the leadership team of the client. At this workshop the revised set of dilemmas was presented. The leadership team also prioritised this new set of dilemmas.

\subsection{Factual findings from research cycle 3}

The factual of findings of this research cycle are summarized in Table 2.

The researcher experienced that it was useful to go through the sequence of SPID per subject or dilemma, resulting in a dilemma phrased in the informant's language. This structure was much better than the ad hoc approach used prior to knowledge of or using SPID. As a consequence, the researcher traversed the SPID sequence several times, generating a series of dilemmas per interview.

In summary, this research cycle reveals improvements in both validity and reliability in the elicitation of dilemmas. Although this cycle has reported the formal investigations during research cycle three, the consultant (and many of his peers) now continue to apply SPID within their ongoing professional practice. Throughout such consulting assignments, there is consistent reliability in dilemmas found and increased client satisfaction through improved validity.

\section{Conclusion}

The main agenda for this research was to explore the dynamics of the elicitation process of dilemmas and if this process could be made more systemized and more rigorous 
Table 2 The factual of findings of this research cycle are summarized in Table 2.

\begin{tabular}{|c|c|c|}
\hline Questions & $\begin{array}{l}\text { Using SPID Consultant } 1 \\
\text { and Researcher }\end{array}$ & Not using SPID Consultant 3 \\
\hline $\begin{array}{l}\text { Consistency between } \\
\text { the results of the } \\
\text { consultants (reliability) }\end{array}$ & $\begin{array}{l}\text { Yes, there is a significant } \\
\text { consistency between the } \\
\text { results of consultant } 1 \text { and } \\
\text { the researcher }\end{array}$ & $\begin{array}{l}\text { No, see research cycle } 1 \mathrm{No} \text {, the } \\
\text { results of consultant } 3 \text { demon- } \\
\text { strate less overlap with the } \\
\text { results of consultant } 1 \text { and the } \\
\text { researcher }\end{array}$ \\
\hline $\begin{array}{l}\text { Client satisfaction } \\
\text { (validity) }\end{array}$ & Yes, broad recognition & $\begin{array}{l}\text { Reasonable, some dilemmas were } \\
\text { recognized others less }\end{array}$ \\
\hline $\begin{array}{l}\text { Important dilemmas not } \\
\text { missed (Validity issues) }\end{array}$ & $\begin{array}{l}\text { According to the client all } \\
\text { important issues were } \\
\text { captured }\end{array}$ & \\
\hline Critical comment & $\begin{array}{l}\text { Both consultant } 1 \text { and the } \\
\text { researcher (consultant 2) } \\
\text { perceived SPID as a } \\
\text { useful tool for eliciting } \\
\text { dilemmas }\end{array}$ & $\begin{array}{l}\text { Consultant } 3 \text { subsequently praised } \\
\text { the work of consultants } 1 \text { and } 2 \\
\text { and the quality of their findings. } \\
\text { Consultant } 3 \text { explained his aim } \\
\text { had been to develop understand- } \\
\text { ing by the client of the value } \\
\text { of thinking in terms of dilemmas, } \\
\text { rather than to elicit the exact and } \\
\text { important dilemmas facing the } \\
\text { client } \sim \text { because even he had no } \\
\text { such means of achieving such a } \\
\text { result }\end{array}$ \\
\hline
\end{tabular}

and located within a theory base-or some new substantive theory constructed. The intention was to inform and improve professional practice and to contribute to theory development in the domain. The purpose of this chapter is to review: (1) a theoretical evaluation of the SPID approach; (2) the aims and primary findings of the research; (3) the contribution to knowledge.

\subsection{A theoretical evaluation of the SPID approach}

This section attempts to construct a theoretical basis for understanding the dynamics that take place when using a framework such as SPID for the elicitation of dilemmas. An attempt is made to integrate and critique the conceptual findings from the three research cycles.

Dilemmaism has been claimed in this paper as a way of consulting (researching). It is a means of challenging assumptions and revealing client's problems (nomothetic and ideographic) based on the forced consideration of opposites. The fundamental issue of trying to elicit the dilemma in the first place has been the focus of this research.

It is therefore now constructive to consider the relationship between the consultant and respondent from the perspective of 'Black Box theory' as conceived in an engineering context in the classic cybernetic treatise by Ashby (1957). His original meta-theoretical position developed for deterministic engineering systems is applied to this research as the basis for this critical evaluation.

\subsubsection{Closed black box}

The 'Problem of the Black Box' arose in electrical engineering. Ashby explains: "The engineer is given a sealed box that has terminals for input, to which he may bring any Springer 
voltages or other disturbances he pleases, and terminals for output, from which he may observe what he can. From his observations, he is to deduce what he can of its contents." (p. 86). In an equivalent manner, the consultant (research cycles 1 and 3 ) is probing a client with questions (inputs) and tries to deduce the problems of his client (in the form of dilemmas) from the corresponding outputs (responses to questions).

By definition, a true 'black box' cannot be opened to look inside and thus no assumptions at all can be made about the nature of the Box and its contents. However, the experimenter can bring or cause a number of inputs and observe the corresponding outputs. By thus acting on the Box, and by allowing the Box to affect him and his recording apparatus, the experimenter is coupling himself to the Box, so that the two together form a system with feedback. Every real system has a large number of possible inputs - of possible means by which the experimenter may exert some action on the Box. Equally, it has a large number of possible outputs - of ways by which it may affect the experimenter as recorded in his observations.

When a generous length of input/output data has been obtained, the experimenter will look for repeating patterns, regularities or repetitiveness in the behavior and try to deduce what is inside the black box in order to either predict outputs for future inputs or (as in cybernetic control) to try to determine what inputs (or combination of inputs) will produce the output desired (goal state).

Ashby (1957) emphasizes that nothing is said about the skill of the experimenter in manipulating the input,-i.e., knowing what inputs to try next. This omission is deliberate, for no skill is required. In pure black box theory we are assuming that nothing is known about the Box, and when this is so, the method of making merely random variations (e.g., guided by throws of a die) on the input questions is just as defensible as any other method, for no facts yet exist that could be appealed to as justification for preferring any particular method. Thus, if faced with a real 'black box,' the consultant would not need or benefit from SPID, because no rationale is required to formulate the questions. The consultant might ask for the birthday of the client, why he chose to wear a green tie, how many miles he drove to the office, etc. Any questions have equal merit when faced with a true black box.

However, unlike the electronics engineer faced with a deterministic closed black box system, the consultant-client interaction is a complex, open, and adaptive system. In particular, and this is the substantive issue, is that the consultant is faced at least with an incompletely observable black box.

\subsubsection{The incompletely observable black box}

Ashby (1957) derived the general rule: "If a determinate system is only partly observable, and thereby becomes (for that observer) not predictable, the observer may be able to restore predictability by taking the past history of the system into account or corresponding behavior previously observed by following a standardized catechism of questions in another identical or equivalent system" (p. 115). Thus, the experience of the consultant, who can recall similar situations by using a structured framework such as SPID with previous clients, can help him/her diagnose the current client's dilemmas.

\subsubsection{Opening the black box}

If the observer can 'open the lid' and see 'inside' the black box, then it becomes a 'white' box and knowledge of the internal workings helps the observer understand 
Fig. 6 Consultant and client shadow map

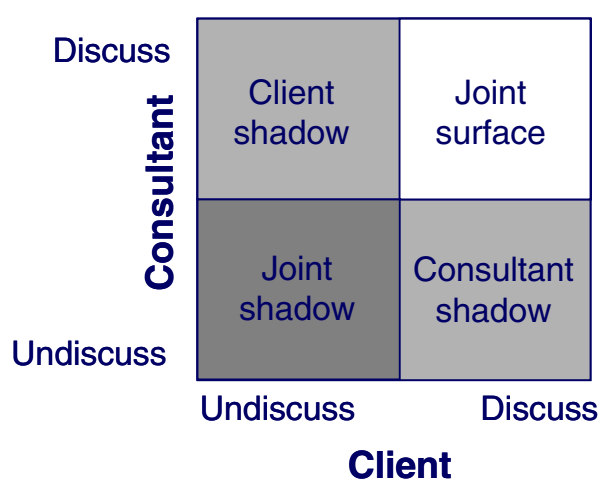

the internal mechanisms. He therefore knows what inputs to apply (questions to ask) in order to generate the output responses required.

The shadow map developed by Cope (2003), which shows significant overlap with the Johari Window that was first used at the Western Training Laboratory in Group Development in 1955 (Luft 1970), is a tool that might be helpful to understand what shadows might reside in the interaction between the consultant and the client. In Figure 8.2 the consultant reflects on any shadow issues in relation to the client. At the surface level the consultant might be prepared to talk about the project plan, background of dilemma reconciliation, etc.

However, shadows for the consultant might be the fact that the project was sold because of pressure from a senior partner to drive up revenue, a concern that the paradigm of inquiry used is not proven, etc.

When the consultant and the client come together a combination of four constructs exists (see Fig. 6):

(1) Joint shadow: the things neither wants to discuss.

(2) Consultant shadow: the things the client will discuss and the consultant will not.

(3) Client shadow: the things the consultant will discuss and the client will not.

(4) Joint surface: the things that both will happily discuss.

The resulting four segments are not fixed elements, since they change in size depending upon the level of disclosure and willingness to share undiscussables by the client and consultant. As both players flex their degree of discussables, so the shape of the shadow map will vary.

Effective clarification can only really take place when the (necessary) shadow has been brought to the surface and both parties are free to understand what is going on, this is what Cope (2003) refers to as 'shadow dancing,' which is the ability to move two key lines within the shadow map, as shown in Fig. 7. Although there will be a variety of strategies that can be used to open up the surface area and shrink the joint shadow area, they will be primary dependent upon the ability to move the two lines in the direction shown by the arrows. The consultant must be able to move their line from top to bottom to make more things discussable and the client must be helped to move the line from right to left and do the same.

Cope (2003) stresses a caution with this model. The goal is to attain the necessary information to facilitate the clarification of a dilemma raised by the client. The Springer 
Fig. 7 Redrawing the shadow map

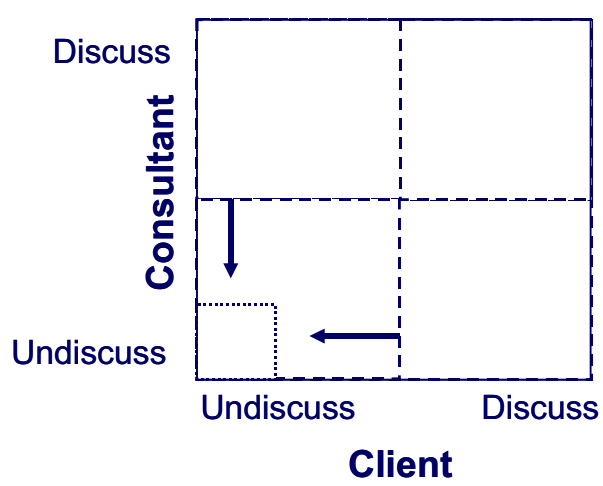

key word is 'necessary.' The danger in surfacing shadows is that all of a sudden the consultant or the client is faced with the undiscussable that neither of party is willing to deal with.

The SPID model as it was tested was only about asking and not so much about telling or disclosing information. According to the Shadow map, one might conjecture that for an effective relationship for eliciting dilemmas, the interviewer should both ask questions about client specific issues and additionally disclose information about dilemmaism.

\subsubsection{The dialectic}

In this 'new' paradigm of inquiry, it has been emphasized that 'dilemmaism' seeks to provide a structured rationale to develop dialectic between the consultant and client. But what we can now conjecture from this discussion of Black Box theory is that:

(a) There are some parallels between the consultant-client interaction (dialectic) and a black box.

(b) The black box is not completely observable - and therefore some of the causeeffect mechanisms inside the black box are unknown (hidden dilemmas?) and the consultant may form an incorrect diagnosis.

(c) The consultant may benefit from previous knowledge (experience) of similar situations to infer unobservable parts of the black box having previously used a framework such as SPID - but this is relative, not absolute.

(d) Unlike a pure black box in which the experimenter needs no skill or strategy, the consultant dealing with an incompletely observable black box can benefit from an experimenting strategy such as SPID that seeks to reveal some of the inside of the black box and offer some white box insight.

(e) The ultimate diagnosis arising from the dialectic-irrespective of the approach adopted by the consultant can never be absolute in the Plato sense because the box always has some unobservable components.

(f) Different consultants (having different degrees of experience, i.e., Ashby memory) may apply different conjectures and thereby still conclude a different diagnosis. 
6.2 Review of the research and reflection on the research questions

The formal research questions, which evolved during the course of the research, were stated as:

(a) What are the dynamics in the process of seeking to explore and elicit dilemmas?

(b) Can the elicitation of dilemmas be made more systematic and rigorous through the development of supporting substantive theory?

Research cycle one sought to explore the first research question. In summary this research cycle demonstrated that when studying the same source material different consultants produce different dilemmas. It was demonstrated that the dynamics of the elicitation process of dilemmas based on current practice is complex. In addition this research cycle showed that the existing methods and approaches used to elicit and capture dilemmas were not reliable or rigorous and that elicited dilemmas too often appear to be invalid.

Reflecting on the second research question it has been demonstrated that the use of SPID, which is developed in research cycle 2, as a framework for electing dilemmas for the practitioner gives added value and some rigor as demonstrated in research cycle 3 . However, there can never be an ultimate framework that is the basis of perfect (professional) practice because the client is always an incompletely observable black box (Ashby 1957). The ultimate diagnosis arising from a dialectic, irrespective of the approach, between the client and the consultant can never be absolute in Plato sense because the box always has some unobservable components. Therefore, different consultants (having different degrees of experience, i.e., Asby's memory) may apply different conjectures and thereby conclude a different diagnosis.

In spite of these limitations, the SPID framework continues to contribute to the professional practice of THT Consulting and to the academic understanding of dilemma theory within dilemmaism. From the practitioner perspective, any improvement itself will be sufficient to justify the research investigation. In addition SPID also serves the basis of accumulating Ashby's memory (1957). Taken all together, it can be claimed that some progress on answering the research questions were realized.

Whilst the research has led to the development of the SPID framework to be used in professional practice, the following sections attempts to generalize this research to a theory domain.

\subsection{Contribution to knowledge and professional practice}

The following contributions to theory and professional practice are claimed:

(1) This research revealed that dilemma theory (Hampden-Turner and Trompenaars 2001) is explored as a paradigm of inquiry that has been named herein as 'dilemmaism.' This opens up a whole range of opportunities to think about different approaches to undertaking research in management and business and other social settings - over and above classical paradigms of inquiry. It challenges conventional wisdom in choices faced by researchers in that choice is no longer limited to positivism, post-positivism, critical theory, constructivism, or participatory paradigms. Dilemmaism might not be as bold as to claim as a paradigm shift, but at least it offers a new (alternative) paradigm with which to view the world. 
(2) A new model to make the process of eliciting dilemmas more rigorous has been developed and validated. This model, called SPID (referring to Situation, Problem, Implication, and Dilemma), is loosely based on Huthwaite SPIN model.

(3) There is evidence that the use of SPID results in a higher degree of reliability and validity in eliciting dilemmas and this makes a contribution to the domain of dilemmaism and the inherent dilemma theory. The prescribed approach is also part of what can also be described as the dilemma methodology component of dilemmaism.

The research thus presents a contribution to both theoretical knowledge and professional practice, one that can be used not only by the researcher but also by my peers within THT Consulting and by other practitioners of dilemmaism.

\section{References}

Arons, B.: Hyperspeech: Navigating in speech-only hypermedia. Hypertext'91, 133-146 (1991)

Ashby, W.R.: An Introduction to Cybernetics. Chapman \& Hall Ltd., London (1957)

Bandler, R. Grinder, J.: Structure of Magic: A Book about Language \& Therapy, Science and Behaviour Books. Palo Alto, CA (1975)

Bateson, G.: Steps to an Ecology of Mind. Ballantine, New York (1972)

Bateson, G.: Mind and Nature: A Necessary Unity. Bantam Books, New York (1976)

Benedict, R.: Patterns of Culture. Houghton, Mifflin Company, Boston (1934)

Berne, E.: Games People Play: The Psychology of Human Relationships. Grove Press, New York (1964)

Blake, R.R., Mouton J.S.: The Managerial Grid III. Gulf Publishing Company, Houston (1985)

Cameron, K., Quinn, R.: Diagnosing and Changing Organisational Culture: Based on the Competing Values Framework. Addison-Wesley Publishing, Reading, MA (1999)

Camus, A.: The Plague (reissued edition, original 1954). Vintage, New York (1991)

Cope, M.: The Seven C's of Consulting: The Definitive Guide to the Consulting Process. FT Prentice Hall, Harlow (2003)

Creswell, J.W.: Qualitative Inquiry and Research Design: Choosing Among Five Traditions. Sage Publications, Thousand Oaks, CA (1998)

Creswell, J.W.: Research Design: Qualitative, Quantitative, and Mixed Method Approaches, 2nd edn. Sage Publications, Thousand Oaks, CA (2003)

Denzin, N.K., Lincoln, Y. (eds.): Handbook of Qualitative Research. Sage Publications, Thousand Oaks, CA (1994)

Durkheim, E.: The Division of Labor in Society. The Free Press, New York (1947)

Easterby-Smith, M., Thorpe, R., Lowe, A.: Management Research-An Introduction. Sage Publications, London, England (1991)

Frankl, V.E.: The Unheard Cry for Meaning. Psychotherapy and Humanism. Simon and Schuster, New York (1978)

Fromm, E. (ed.): 2000, The Art of Loving, Perennial. London (1956)

Gill, J., Johnson, P.: Disruptive change: when trying harder is part of the problem. Harvard Business Rev. 80(5), 3-8 (2002)

Hampden-Turner, C.: Interviewing to elicit dilemmas. Internal paper (1999)

Hampden-Turner, C., Trompenaars, F.: Building Cross-Cultural Competence: How to create wealth from conflicting values. Wiley Ltd., Chisester (2000)

Handy, C.: The Age of Unreason. Harvard Business School Press, Cambridge, MA (1990)

Hunt, S.: 1991, Positivism and paradigm dominance in consumer research: toward critical pluralism and rapprochement. J. Consumer Res. 18(1), 32-44 (1991)

Heron, J., Reason, P.: A participatory inquiry paradigm. Qualitative Inquiry, 3, 274-294 (1997)

Hussey, J., Hussey, R.: Business Research - A Practical Guide for Undergraduate and Postgraduate Students. MacMillan Press, Hampshire, England (1997)

Johnson, B.: 1992, Polarity Management, Identifying and Managing Unsolvable Problems, HRD Press, Amherst, Massachusetts.

Jung, C.G. In: The portable Jung. In: Campbell. J. (ed.) Viking Penguin, New York (1971) 
Kant, E.: Critique of Pure Reason. Trans. W. Pluhar, Hackett, Indianapolis (1996)

Kuhn, T.: 1970, The Structure of Scientific Revolutions, 2nd edn. University of Chicago Press, Chicago, IL (1970)

Lévi-Strauss, C.: Structural Anthropology. Basic Books, New York (1963)

Luft, J.: Group processes; an introduction to group dynamics, 2nd edn. National Press Books, Palo Alto, CA (1970)

Maslow, A.: Motivation and Personality. Harper, New York (1954)

Mason, J.: Qualitative Researching. Sage Publications, London, England (1996)

May, R.: Psychology and the Human Dilemma. Van Nostrand Reinhold, New York (1967)

McGregor, D.: The Human Side of Enterprise. Penguin, London (1987)

Mintzberg, H.: The Rise and Fall of Strategic Planning: Reconceiving Roles for Planning, Plans, Planners. Free Press, New York (1994)

Neuman, W.L.: Social Research Methods: Qualitative \& Quantitative Approaches. Allyn \& Bacon, Needham Heights (1997)

Newman, I., Benz, C.R.: Qualitative-Quantitative Research Methodology: Exploring the Interactive Continuum. Southern Illinois University Press, Carbondale (1998)

Porter, M.E.: Competitive Strategy, Techniques for Analyzing Industries and Competitors. The Free Press, New York (1980)

Quinn, R.E., Cameron, K.S.: Organisational paradox and Transformation. In: Quinn, R.E., Cameron, K.S. (eds.) Paradox and Transformation: Toward a Theory of Change in Organisation and Management, pp. 12-18. Ballinger, Cambridge, MA (1988)

Rackham, N.: Spin Selling. McGraw-Hill, New York (1988)

Russell, B.: My Philosophical Development. Routledge, London and New York (1995)(1959)

Sartre, J.P.: Being and Nothingness. Washington Square Press, Washington, DC (1993)

de Saussure, F.: Course in General Linguistics. Translated from the French by W. Baskin, London (1960)

Seale, C.: Researching Society and Culture. Sage Publications, London (1998)

Silverman, D.: Interpreting Qualitative Data: Methods for Analysing Talk, Text and Interaction. Sage Publications Ltd., UK (1993)

Trist, E.L., Emery, F.E.: The Social Engagement of Social Science: A Tavistock Anthology. University of Pennsylvania Press, Philadelphia, PA (1990)

Trompenaars, F., Hampden-Turner, C.: Riding the Waves of Culture: Understanding Cultural Diversity in Business. Nicholas Brealey Publishing, London (1998)

Trompenaars, F., Hampden-Turner, C.: 21 Leaders for the 21st Century: How Innovative Leaders Manage in the Digital Age. Capstone Publishing Ltd, Oxford (2001)

Trompenaars, F., Woolliams, P.: Business Across Cultures. Capestone Publishing Ltd., Chichester, England (2003)

de Vries, K.M.F.R.: Organisational Paradoxes: Clinical Approaches to Management. Routledge, New York (1995)

Watt, S.L.B.: Probing Linkages between Innovation and Corporate Culture. Unpublished doctoral thesis, La Trobe University, Bundoora, Victoria, Australia (2002)

de Wit, B., Meyer, R.: Strategy Synthesis: Resolving Strategy Paradoxes to Create Competitive Advantage. Thomson Learning, London (2000)

Wolcott, H.F.: 1975, Criteria for an ethnographic approach to research in schools. Human Organisations, 34, 111-127 (1975) 